\title{
Accuracy Improvement of a Photoacoustic Helmholtz Cell Model
}

\author{
M. SUCHENEK* AND L.J. OPALSKI \\ Institute of Electronic Systems, Warsaw University of Technology, Nowowiejska 15/19, 00-665 Warsaw, Poland \\ (Received November 6, 2013; revised version March 6, 2014; in final form March 13, 2014)
}

This paper presents a method for improving accuracy of the frequency response of a photoacoustic Helmholtz cell model. The method generates simple non-linear corrections to well-established (reference) delay line model of the cell. The form of the correction functions is obtained from a study of a numerical fitting of the reference model to the measurement data. A novel integral accuracy measure is formulated to compare fit of cell models to actual measurements in the neighborhood of the resonance frequency, which is important for measurement applications of the cell. The two proposed correction functions modify effective values of duct parameters. Despite simplicity the proposed corrections make significant improvement of modeling accuracy, as shown with the integral measure but also with prediction accuracy of the resonance frequency and the cell quality factor. It is significant that substantial accuracy gain due to the corrections was confirmed with measurements of a second (testing) set of photoacoustic Helmholtz cells with geometrical parameter values which are different from these of the first set.

DOI: $10.12693 /$ APhysPolA.125.1132

PACS: 43.20.Ks, 43.20.Wd, 43.30.Zk, 43.38.Zp

\section{Introduction}

Measurement of photoacoustic signal is quite a challenging task [1, 2], especially in case of small amounts of substance under test $[3,4]$, and also for substances with a very weak absorbing spectrum. For such measurements acoustic response can be extremely small and furthermore it can be covered with measurement equipment noise and interference from external acoustic signals. Measurement conditions can improve significantly if the tested substance can be placed inside the acoustic cell. Such an arrangement inter alia separates measured sample from the external optical and acoustic interference sources and so it increases signal to noise ratio. Additionally, a sample might be excited periodically by the modulated light source. If the excitation frequency is equal to the cell resonance frequency - the signal due to the acoustic response of the sample is emphasized, while all stray signal components, including noise at frequencies which are distant from the resonance, are suppressed. That kind of selectivity makes possible extraction of the response from absorbing material even in the presence of strong background signals.

For this reason it is important to design acoustic cells with resonant parameters (i.e. the resonance frequency and selectivity) which are appropriate for particular measurement needs. Accurate modeling of cells with different size and shapes [5] is also important, as it enables design and implementation of the appropriate cell on the first attempt - without costly and time consuming trials of several physical implementations and subsequent selection of the most appropriate cell based on measurements.

*corresponding author; e-mail: M.Suchenek@ise.pw.edu.pl
In the literature different photoacoustic cell designs can be found [6-9]. Recently cells exhibiting Helmholtz resonance become more and more popular [10, 11], as they offer both low resonant frequencies as well as small geometrical dimensions.

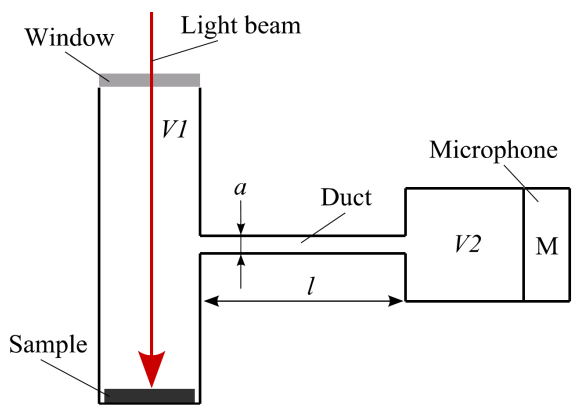

Fig. 1. A schematic layout of the photoacoustic Helmholtz resonator.

Figure 1 shows an example design of such a cell - two cavities (with volumes $V 1$ and $V 2$ ) connected by the duct with length $l$ and diameter $a$. The sample is excited by a modulated light beam in one cavity, while sound waves are measured in the other cavity by the microphone $M$.

\section{Photoacoustic Helmholtz cell model}

The Helmholtz cells for photoacoustic applications can be modeled by acousto-electrical analogies. The acoustic response of the light-excited sample is represented as a periodic current source and each cavity is represented as a capacitor. The cell models differ mainly in how they represent the duct which connects the cavities [12-17]. According to the study of the photoacoustic Helmholtz resonator models [18] the best agreement between models 
and measurement data can be found for models which represent the duct with delay line or with lumped RLC elements as defined by Kastle and Sigrist [12].

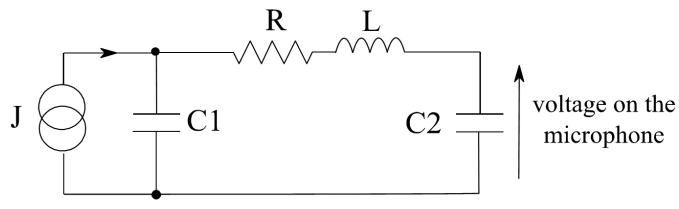

Fig. 2. Helmholtz resonator model with lumped elements.

If the cell dimensions are much smaller than the acoustic wave length used, the duct should be modeled with lumped elements, as shown in Fig. 2. The current source $J$ represents the excitation of the test sample by the modulated light beam, while capacitors $C 1$ and $C 2$ represent respectively measurement cavity (in which the test sample is placed) and the microphone cavity. The duct is represented by an inductance $L$ and the resistance $R$.

Each element of the model is best described by formulae proposed by the Kastle and Sigrist [12]:

$$
R=\frac{\rho l \omega\left[d_{\mathrm{v}}+(\gamma-1) d_{\mathrm{t}}\right]}{\pi a^{3}}, \quad L=\frac{\rho l}{\pi a^{2}}, \quad C_{i}=\frac{V_{i}}{\rho u^{2}},
$$

where $\rho$ is described as the mass density of the gas, $u$ is the speed of the sound in free space, $a$ is the diameter of the duct, $l$ is the length of the duct, $V_{i}, i=1,2$ are volumes of the measurement and the microphone cavities, respectively. $\gamma=C_{p} / C_{v}$ is the ratio of the specific heats at constant pressure and volume, $d_{\mathrm{v}}$ and $d_{\mathrm{t}}$ are thickness of viscous and thermal boundary layer

$$
d_{\mathrm{v}}=\sqrt{\frac{2 \mu}{\rho \omega}}, \quad d_{\mathrm{t}}=\sqrt{\frac{2 \kappa}{\rho \omega c_{p}}},
$$

$\mu$ denotes viscosity of the gas filling the cell, $\kappa$ is the heat conductivity, $\omega=2 \pi f, f-$ modulation frequency.

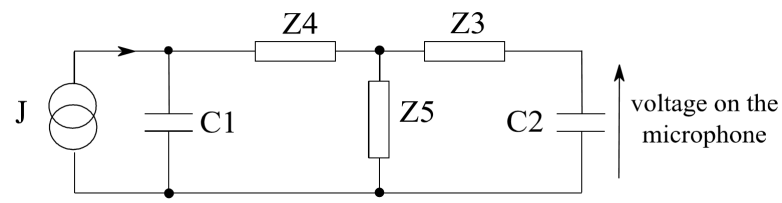

Fig. 3. Helmholtz resonator model with the transmission line changed to the T-circuits.

If the cell dimensions are comparable to the acoustic wave length used, the duct should be modeled with a transmission (delay) line. The model can be drawn as shown in Fig. 3 - if the delay line is replaced by the T-circuit, consisting of appropriately defined three impedances $Z 3, Z 4$, and $Z 5$ :

$$
Z_{3}=Z_{4}=Z_{w} \tanh \left(\frac{\Gamma_{w} l}{2}\right), Z_{5}=\frac{Z_{w}}{\tanh \left(\Gamma_{w} l\right)} .
$$

The characteristic impedance $Z_{w}$ and the propagation constant $\Gamma_{w}$ of this model were described by Benade [19] and Daniels [20, 21] as

$$
\begin{aligned}
Z_{w} & =\sqrt{\frac{R+\mathrm{i} \omega L}{G+\mathrm{i} \omega C}}, \\
\Gamma_{w} & =\sqrt{(R+\mathrm{i} \omega L)(G+\mathrm{i} \omega C)},
\end{aligned}
$$

and $R, G, L, C$ were described as

$$
\begin{aligned}
& R=-\frac{\omega \rho}{\pi a^{2} D^{2}} F_{\mathrm{v}} \sin \left(\Theta_{\mathrm{v}}\right), \\
& L=\frac{\rho}{\pi a^{2} D^{2}}\left(1-F_{\mathrm{v}} \cos \left(\Theta_{\mathrm{v}}\right)\right), \\
& G=-\frac{\omega \pi a^{2}}{\rho u^{2}}\left[(\gamma-1) F_{\mathrm{t}} \sin \left(\Theta_{\mathrm{t}}\right)\right], \\
& C=\frac{\pi a^{2}}{\rho u^{2}}\left[1+(\gamma-1) F_{\mathrm{t}} \cos \left(\Theta_{\mathrm{t}}\right)\right], \\
& F_{x} \exp (\mathrm{i} \Theta)=\frac{2 J_{1}\left((-1)^{0.5} r_{x}\right)}{(-1)^{0.5} r_{x} J_{0}\left((-1)^{0.5} r_{x}\right)}, \\
& D^{2}=\left(F_{\mathrm{v}} \sin \Theta_{\mathrm{v}}\right)^{2}+\left(1-F_{\mathrm{v}} \cos \Theta_{\mathrm{v}}\right)^{2}, \\
& r_{\mathrm{v}}=a\left(\frac{\omega \rho}{\mu}\right)^{0.5}, \quad r_{\mathrm{t}}=a\left(\frac{\omega \rho c_{p}}{\lambda}\right)^{0.5} .
\end{aligned}
$$

Unfortunately, definitions of the Helmholtz cell model parameters, which can be found in the literature, do not lead to good agreement of cell response predictions and actual responses [22-24]. We will show that accuracy of existing cell models can be significantly improved with simple non-linear correction functions. The accuracy improvement will be demonstrated for the delay line based model.

\section{Design and measurement of photoacoustic Helmholtz cells}

Development and verification of the cell model improvement technique, to be presented in the next sections, was possible due to availability of a substantial number of the Helmholtz cells with different geometrical parameter values and common mechanical design - as presented in Fig. 4. Elements of these cells were designed in such a way that the cells can be easily reassembled by using components of different sizes. Each element (microphone, sample cavity, duct connecting cavities) can be easily replaced for another instance of that element (possibly with different size).

Having that flexibility we created two sets of cell parameter values. For the first set we used combinations of: the volume of the microphone cavity $V 2=$ $0.5,1.0,1.5,2.0 \mathrm{~cm}^{3}$ (the measurement cavity was fixed at $V 1=2 \mathrm{~cm}^{3}$ ), the duct length $l=2,3,4 \mathrm{~cm}$ and the diameter $a=1,3 \mathrm{~mm}$. The second parameter set was formed with a combination of: the volume of the microphone cavity $V 2=1.0,2.0,3.0,4.0 \mathrm{~cm}^{3}$ and measurement cavity $V 1=1.0,2.0,3.0,4.0 \mathrm{~cm}^{3}$, the duct length $l=1.5,2.5,3.5,4.5 \mathrm{~cm}$ and the diameter $a=2,4 \mathrm{~mm}$. 


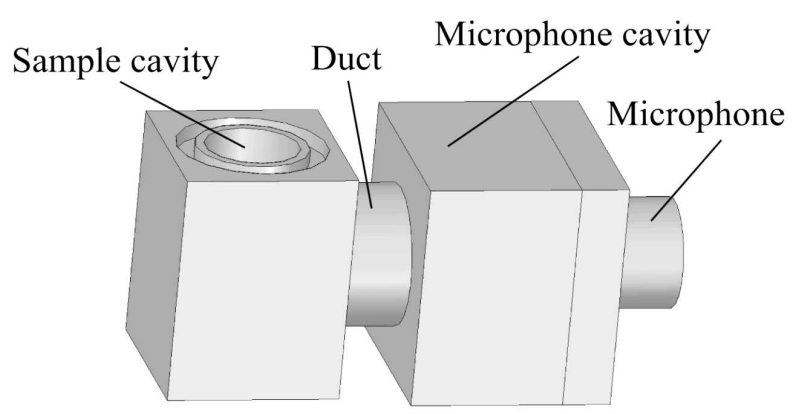

Fig. 4. Mechanical construction of photoacoustic Helmholtz cell.

The first set was used to obtain the best form of correction functions for the delay line based model and the second one - to evaluate the proposed model modifications.

All mechanical components were made of brass in order to ensure appropriate stiffness of the whole cell and eliminate vibrations. Prior to measurements the cell was placed in the holder, as shown in Fig. 5.

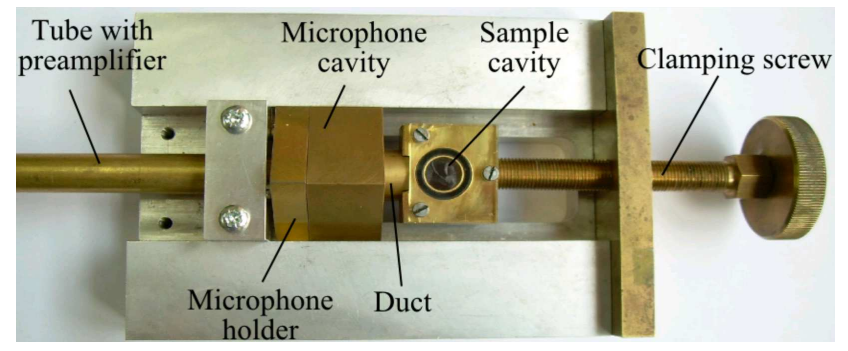

Fig. 5. Mechanical construction of photoacoustic Helmholtz cell.

The step-byśtep method [25] was used to obtain frequency responses of the cells in the frequency range $200 \mathrm{~Hz}$ to $4 \mathrm{kHz}$. Thus the sample cell was stimulated by a light beam from a LED source, modulated at one frequency at a time. The photoacoustic signal was collected by a capacitive microphone. Electric signal was amplified 100 times with a shielded preamplifier before it was digitally recorded. Measured signal was relatively low, and so required an additional averaging. Steady-state signal amplitude at resonance frequency was in order of few millivolts after about 1000 times averaging.

\section{Accuracy of the reference model for Helmholtz cell models}

Before any discussion of model accuracy improvement it is necessary to understand what accuracy means for a particular application of the model, and to formulate the model accuracy measures. For Helmholtz cells under scrutiny the most interesting center parts of measured and predicted frequency responses can have the form as shown in Fig. 6.

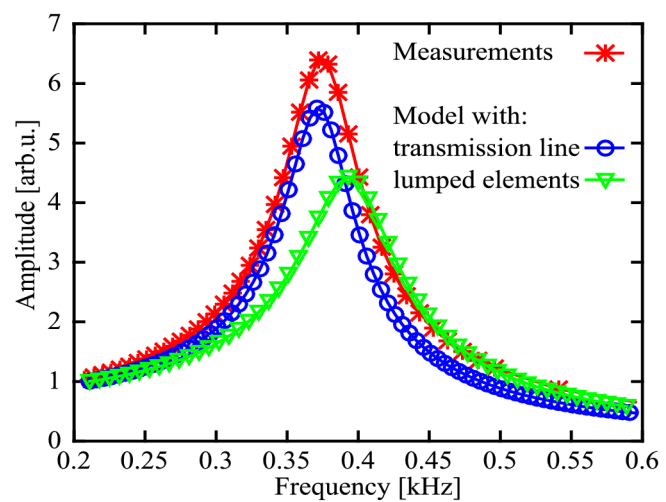

Fig. 6. Frequency response from photoacoustic Helmholtz cell compared with model with lumped elements and transmission line. For the cell with sample volume $4 \mathrm{~cm}^{3}$, microphone $2 \mathrm{~cm}^{3}$, duct length $45 \mathrm{~mm}$ and diameter $2 \mathrm{~mm}$.

For photoacoustic applications it is reasonable to consider distance between the model predicted response signal $U_{\mathrm{m}}(f, \boldsymbol{p})$ and the actual measurement $U_{\mathrm{d}}(f, \boldsymbol{p})$ for the sinusoidal excitation of the frequency $f$ in a close neighborhood of the resonance frequency $f_{0}$ ( $\boldsymbol{p}$ denotes a vector of designable cell parameters, such as volumes of cavities, length and diameter of the duct). In this work it was assumed that the endpoints $f_{1}$ and $f_{2}$ of the frequency range of interest are the frequencies at which the amplitude of the response signal drops $3 \mathrm{~dB}$ from the maximum amplitude value. Subsequently the quality factor of the cell was defined as $Q=f_{0} /\left(f_{2}-f_{1}\right)-$ by analogy to the widespread definition of the $Q$-factor for RLC tanks.

The important point of our approach is that we do not use direct comparison of the resonant frequency $f_{0}$ and the $Q$-factor of a particular cell and its model to assess quality of a model. Instead we propose using the following integral model accuracy (IMA) measure:

$$
\delta(\boldsymbol{p})=\frac{1}{f_{2}-f_{1}} \int_{f_{1}}^{f_{2}}\left|U_{\mathrm{m}}(f, \boldsymbol{p})-U_{\mathrm{d}}(f, \boldsymbol{p})\right| \mathrm{d} f .
$$

The frequency domain response signal $U_{\mathrm{m}}(f, \boldsymbol{p})$ of cells used in our study was modeled with the circuit shown in Fig. 3. In the actual calculation of the IMA measure the resonance frequency $f_{0}$ and frequencies $f_{1}$ and $f_{2}$ were determined by the spline approximation of cell responses, and the value of the integral accuracy measure was calculated by the trapezoidal quadrature.

At the first stage of our model accuracy improvement procedure we made a numerical minimization of the IMA measures $\delta^{(i)}(\boldsymbol{p}), i=1, \ldots, N$ formulated separately for each of $N=24$ cells from the first set. That way three components of the $\boldsymbol{p}$ vector represented the duct length $l$ and diameter $a$, the volume of cavity $V 2$. Let us denote optimum design parameters with $\boldsymbol{p}_{\mathrm{m}}^{(i)}, i=1, \ldots, N$ :

$$
\boldsymbol{p}_{\mathrm{m}}^{(i)}=\min _{\boldsymbol{p}} \delta^{(i)}(\boldsymbol{p}) \text {. }
$$


In our case the vector $\boldsymbol{p}_{\mathrm{m}}^{(i)}$ contains 3 optimum model parameter values, referred to later as $l m, a m, V 2 m$. Geometrical dimensions of the cell were used as a starting point of the iterative numerical minimization algorithm fmincon of the MATLAB optimization toolbox [26]. For each optimum vector we recorded corresponding value of the IMA measure: $\delta_{\mathrm{m}}^{(i)} \equiv \delta\left(\boldsymbol{p}_{\mathrm{m}}^{(i)}\right)$ to control numerical quality of the best fit and so to detect possible failures of the optimization based fitting.

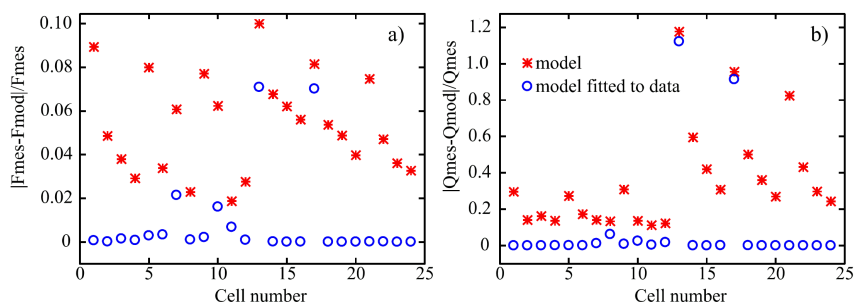

Fig. 7. Disagreement between models, model numerical fitted and measured data as expressed by (a) resonance frequency, (b) quality factor. Fmes, Qmes - resonance frequency and quality factor calculated from measurement data; Fmod, Qmod - resonance frequency and quality factor calculated from the model.

A useful side-effect of IMA calculations was calculation of the resonant frequency $f_{0}$ and the quality factor $Q$ for each cell model. That way it was possible to verify not only optimization-based improvement of the IMA measure, but also more meaningful features of the model, i.e. the resonant frequency $f_{0}$ and the quality factor $Q$. For the first set of cells it turned out that the average relative disagreement between the fitted model and measurement of the $Q$-factor was only $8.75 \%$ and $0.83 \%$ for $f_{0}$. Responses of three cells turned out to be fitted poorly, as can be also seen in Fig. 7. Thorough check of measurement procedure for the three cells confirmed that such cells, with large volume of channel (relative to volume $V 2$ ), are indeed modeled so inaccurately that fitting is not able to improve accuracy of $f_{0}$ and $Q$ predictions much. If the three data points are omitted, the average error of the $Q$-factor is reduced to impressive $0.35 \%$ and the error of resonant frequency to $0.1 \%$.

If the original, i.e. geometric values of cell parameters, were used with the same model and the same cell parameters the average relative error for the $Q$-factor was as large as $35.5 \%$ and for $f_{0}$ it was $5.37 \%$.

If it was possible to determine values: $l m, a m, V 2 m$ for each cell instance without numerical optimization, but only via some algebraic transformations of the geometrical parameters of the cell $\boldsymbol{p}=[l, a, V 2]$ - the accuracy predictions of the two important parameters $\left(f_{0}, Q\right)$ of a photoacoustic cell could be much improved.

We cannot hope to formulate such an ideal transformation of $\boldsymbol{p} \rightarrow \boldsymbol{p}_{\mathrm{m}}$ which would be exact for all $N$ pairs of vectors $\boldsymbol{p}^{(i)} ; \boldsymbol{p}_{\mathrm{m}}^{(i)}$. Instead we will show that it was possible to construct a simple algebraic transformation of the geometrical parameters into the model parameters which retains quite much of accuracy gain of the ideal transformation.

In order to reduce the complexity of the new model, we were looking for a minimum subset of the designable parameters which would give substantial improvement of the modeling accuracy. It was found that the biggest influence has the duct size: diameter $a$ and length $l$. Minimization of IMA measures with respect to (w.r.t.) only two of these parameters for the first set of cells resulted in decrease of the average error of the $Q$-factor to $13.8 \%$ and for the resonant frequency $f_{0}$ to $1.15 \%$. Removing again the three worst fitted data points, resulted in decrease of the average error of the $Q$-factor to $0.31 \%$ and resonant frequency to $0.62 \%$. Further attempts to reduce the number of parameters, e.g. to the diameter or only to the duct length, did not result in a significant reduction of the modeling error. Similar experiments for the second set of cells confirmed the largest influence of the duct size upon modeling accuracy of single cells.

\section{Correction function for the model with the transmission line}

In the next step of our model improvement method we were trying to find two functions which would map the physical dimensions of the duct $l$ and $a$ to values close to these obtained numerically, i.e. $l m$ and $a m$. Finding correct mappings were done in two sub-steps. First, simple parameterized functions were discovered, and then values of the parameters were determined, good for the whole population of cells from the first set. After that quality of the mapping functions was tested using an independent (second) set of (testing) cells.

To discover functional form of the mappings we used results of fitting model responses to measurements for 24 cells of the first set, in fact the 24 pairs of values: $l m$, and $a m$ which correspond to physical duct sizes of the 24 cell instances, i.e. length $l$, diameter $a$. The relations between the geometric dimensions and corresponding numerically fitted values of the duct dimensions are shown in Fig. 8.
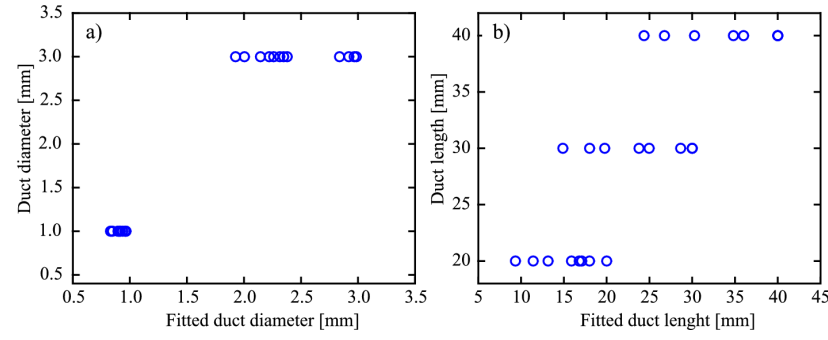

Fig. 8. Relations between physical duct dimensions and obtained numerical values duct (a) diameter, (b) length.

From the figures we can see that better agreement between model and measured data could be obtained if the dimension of the duct used for the model calculations was 
smaller than the geometric dimension of the corresponding cell. This remark seems to be true for all dimensions of the duct in the model.

We started our quest for good mappings, $l$ to $l m$, and $a$ to $a m$, from linear functions, to discover that modeling accuracy could be slightly improved. Much better results were obtained with nonlinear functions. Many trials were made with functions involving not only the duct length and diameter, but also volumes of each cavities and the duct and their inverses. The following functions were finally selected - as a compromise between simplicity and accuracy improvement

$$
l_{p}=\frac{l}{c_{1}+c_{2} a}, \quad a_{p}=\frac{a}{c_{3}+c_{4} a},
$$

where $l_{p}$ approximates $l m$ and $a_{p}$ approximates am values. For clarity let us add that the four constants $c_{1}, \ldots, c_{4}$, which appear in the last formulae, were obtained by optimization-based tuning. The tuning was set up as minimization of the sum of IMA values for all 24 cell models considered in the first set of cell parameters w.r.t. the four coefficient values. Resulting mapping were found as follows:

$$
\begin{aligned}
& l_{p}=\frac{l}{0.861987+a 0.304575}, \\
& a_{p}=\frac{a}{0.973093+a 0.141525} .
\end{aligned}
$$

To validate corrected model, i.e. the delay line based cell model for which dimensions of the duct were calculated by the transformation functions, the second (testing) set of cells was used. Predicted $Q$ and $f_{0}$ values for 128 instances of the Helmholtz cell of the second set, and corresponding predictions of the model are shown in Fig. 9.
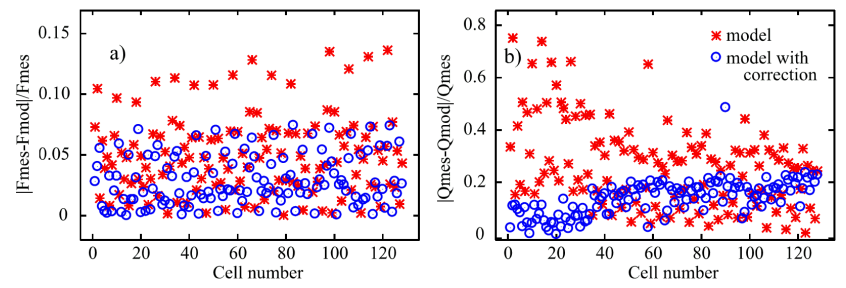

Fig. 9. Disagreement between the original model, model with numerically fitted coefficients, model with correction function and measured data as represented by (a) resonance frequency, (b) quality. Fmes, Qmes - resonance frequency and quality factor calculated from measurement data; Fmod, Qmod - resonance frequency and quality factor calculated from model.

It is seen that the proposed simple nonlinear correction functions, which were developed for one set of cells, enabled significant improvement in the quality of the modeling also for another set of cells (the model without correction is shown with red asterisks while the model with the proposed two non-linear correction functions is shown with blue circles). Figure 10 demonstrates the accuracy improvement perhaps more vividly — showing correlations between measurements and model predictions. For the ideal model the markers should lay along a line inclined at $45^{\circ}$.
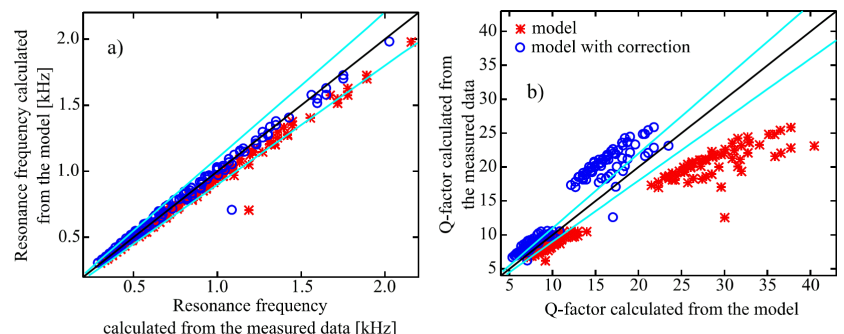

Fig. 10. Correlation between fitted and measured values of (a) the resonant frequency $f_{0}$, (b) the $Q$-factor. The black line denotes the ideal correlation, while the blue lines $\pm 10 \%$ relative difference of the corresponding values.

For the majority of measurement data points of the second set, the model with the proposed correction provides a better prediction of the resonant frequency, and quality factor than the correction-less model. The average error of the quality factor $Q$ was $10.9 \%$ as compared to $35.5 \%$ for the reference model. For the resonant frequency $f_{0}$ the average error is $2.17 \%$ while for the model without correction $5.37 \%$. These results should be considered good, if we realize that the model allows for a good prediction accuracy of main photoacoustic cell parameters in a wide range of resonance frequency and quality factor values, and that properties of the second (testing) set were significantly different from these of the first set. The two sets of cells did not have a common subset of cell parameter values. Additionally, the volume $V 1$ of the cells in the second set was variable, while it was fixed for the cells of the first set.

Since the coefficients values were obtained by fitting the model to the specific measurement data obtained from available cells, for cells with significantly different dimensions or layout further recalibration of the model and recalculation of the coefficients might be needed. Since the correction functions depend only on four numeric coefficients - full recalibration could be possible with only four cell measurements - if the measurement inaccuracy and cell manufacturing inaccuracy could be neglected. If these inaccuracies were significant, a regression type of the recalibration would be necessary, with increase in the number of cells required to improve outcome of the recalibration process.

\section{Conclusions}

This paper presents a method for improving the accuracy of the photoacoustic Helmholtz cell model with transmission line. The improvement is based on introduction of additional correction functions which relate geometrical parameters of the cell with the model input 
parameters. The form of the correction functions was obtained from a study of a numerical fitting of a reference model to the measurement data for the first set of 24 cells with different sizes. Four parameters of the correction function were determined by fitting the corrected model to responses of all 24 cells at the same time. Subsequently the correction functions were verified with another set of 128 cells. It turned out that the significant improvement was achieved by introducing two simple nonlinear correction functions of the duct size, each function is dependent on only two coefficients.

\section{References}

[1] J. Davidsson, J.H. Gutow, R.N. Zare, J. Phys. Chem. 94, 4069 (1990).

[2] S. Danworaphong, I.G. Calasso, A. Beveridge, G.J. Diebold, C. Gmachl, F. Capasso, D.L. Sivco, A.Y. Cho, Appl. Opt. 42, 5561 (2003).

[3] J.-P. Besson, S. Schilt, E. Rochat, L. Thevenaz, Appl. Phys. B 85, 332 (2006).

[4] A. Elia, P.M. Lugarà, C. Di Franco, V. Spagnolo, Sensors 9, 9616 (2009).

[5] M. Suchenek, Int. J. Thermophys. 32, 886 (2011).

[6] V. Gorelik, V.S. Starovoitov, Opt. Spectrosc. 107, 830 (2009).

[7] A. Miklós, P. Hess, Z. Bozóki, Rev. Sci. Instrum 72, 1937 (2001).

[8] J.M. Rey, D. Marinov, D.E. Vogler, M.W. Sigrist, Appl. Phys. B 80, 261 (2005).

[9] F.G.C. Bijnen, J. Reuss, F.J.M. Harren, Rev. Sci. Instrum. 67, 2914 (1996).

[10] S. Barbieri, J.-P. Pellaux, E. Studemann, D. Rosset, Rev. Sci. Instrum. 73, 2458 (2002).

[11] J. Zhao, Z. Zhao, L. Du, S. Wu, Appl. Opt. 50, 4936 (2011).

[12] R. Kastle, M.W. Sigrist, Appl. Phys. B 63, 389 (1996).

[13] O. Nordhaus, J. Pelzl, Appl. Phys. 25, 221 (1981).

[14] J. Blitz, Elements of Acoustic, Butterworths, London 1964.

[15] M. Mattiello, M. Nikles, S. Schilt, L. Thevenaz, A. Salhi, D. Bart, Y. Rouillard, R. Werner, J. Koeth, Spectrochim. Acta A: Mol. Biomol. Spectrosc. 63, 952 (2006).

[16] P.M. Morse, Vibration and Sound, McGraw-Hill, New York 1948.

[17] A.W. Nolle, J. Acoust. Soc. Am. 25, 32 (1953).

[18] M. Suchenek, Proc. SPIE 6937, 6937 (2008).

[19] A.H. Benade, J. Acoust. Soc. Am. 44, 616 (1968).

[20] F.B. Daniels, J. Acoust. Soc. Am. 22, 563 (1950).

[21] F.B. Daniels, J. Acoust. Soc. Am. 19, 569 (1947).

[22] T. Starecki, J. Acoust. Soc. Am. 122, 2118 (2007).

[23] J. Pelzl, K. Klein, O. Nordhaus, Appl. Opt. 21, 94 (1982).

[24] S. Sang-Hyun, K. Yang-Hann, J. Acoust. Soc. Am. 118, 2332 (2005).
[25] M. Suchenek, T. Starecki, Int. J. Thermophys. 32, 893 (2011).

[26] The MATLAB optimization toolbox user's guide: www.mathworks.com/help/pdf_doc/optim/ optim tb.pdf . 